\title{
An Integrated Method for Three-phase AC Excitation and High-frequency Voltage Signal Injection for Sensorless Starting of Aircraft Starter/Generator
}

\author{
Jiadan Wei, Member, IEEE, Hua Xu, Bo Zhou, Zhuoran Zhang, Senior Member, Chris Gerada, Senior Member
}

\begin{abstract}
This paper proposes an integrated method of three-phase AC excitation and high frequency voltage signal injection (HFVSI) for sensorless controlled starting of brushless synchronous machines (BSM) used as starter/generator in variable frequency $A C$ power systems of civil aircraft. Fixed $400 \mathrm{~Hz}$ of the three-phase AC power is adopted both for the AC excitation and HFVSI in the initial starting process to eliminate the bulky rotor position sensor for BSM. The resulting $6^{\text {th }}$ sequence harmonic voltage determined by the rotating rectifier is utilized as the HFVSI into the field-winding of main generator without any extra high frequency signals injection. The rotor position is estimated by the high frequency response signals extracted from the armature windings of main generator. Due to the nonlinear rotating rectifier linked in the HFSI chain, a novel frequency-insensitive asynchronous demodulation strategy is proposed in this paper for rotor position estimation. Furthermore, the initial rotor position detection is calibrated by polarity decision of the induced currents of the armature windings within the establishment procedure of field current of the main generator at standstill. The effectiveness of the AC excitation and feasibility of rotor position estimation for sensorless starting control of BSM are validated by the simulation and experimental results.
\end{abstract}

Index Terms-sensorless controlled starting, brushless synchronous machine (BSM), asynchronous demodulation strategy, initial rotor position detection.

\section{INTRODUCTION}

$\mathrm{D}$ ue to the mature structure, high reliability and low maintenance, the brushless synchronous machine(BSM) is still the most attractive candidate generator topology for both fixed-frequency and variable-frequency $\mathrm{AC}$ power generation for civil aircraft. It has also the potential to be used as the starter for engine cranking[1-3]. In the generation mode, the $\mathrm{AC}$ output of the main exciter of BSM is rectified by a rotating

Manuscript received April 14, 2018; revised July 23, 2018; accepted September 5, 2018. This work was supported by the National Nature Science Foundation of China under Projects 5187110 and 51737006, and the Fundamental Research Funds for the Central Universities XCA17003-01.

The authors are with the Center for More Electric Aircraft Power System(CMEAPS), College of Automation Engineering, Nanjing University of Aeronautics and Astronautics, Nanjing, 211106, China (e-mail:weijiadan@nuaa.edu.cn). rectifier and employed for the DC excitation of main generator. The output voltage magnitude can then be regulated through an automatic voltage regulator to compensate for the variable speed and changing load. However, in the starting mode, the main exciter can't offer excitation power for the main generator from standstill to the low speed range by the conventional DC excitation method. Thus, excitation becomes the first key issue of BSM used as starter in AC variable frequency power system[4].

The usual approach is to adopt a single-phase AC excitation for the stator windings of the main exciter directly, the limited excitation power and side effect of the high inductance of the field winding will further deteriorate the excitation effect and the starting performance. Moreover, this method is unlikely to be suitable for high-power BSM used as a starter for cranking commercial engine in variable frequency AC power system [5]. Literature [6-9] reports different methods to circumvent this. [6] presented a novel structure of the main exciter with additional three-phase ac excitation windings for the AC excitation strategy used in the initial start until the engine approaches a certain speed. [7] adopted an independent three-phase excitation windings with a novel AC excitation method during the whole process of start and generation modes. [8] and [9] proposed a new two-phase symmetrical winding concept for the exciter for constant excitation control of the main generator. This kind of field winding arrangement can be considered as an equivalent to a balanced 3-phase winding set. The reconfiguration of three-phase excitation winding of the main exciter has up till now been the method of choice to enable BSM to be used as a starter for engine cranking.

Furthermore, an extra rotor position sensor capable of tolerating the harsh conditions, such as rotary transformer, has to be assembled in the BSM for the rotor position detection in the starting control of the aircraft application. This additional rotary transformer leads to undesirable additional bulk mass, high cost and a more complex structure. Moreover, the rotor position sensor is only used in the starting procedure, the utilization rate is very low since BSM is normally working in generator mode. Hence, a sensorless starting control strategy eliminating the bulky rotor position sensor is a highly desirable solution of BSM used as the aircraft starter/generator.

In recent literatures, the sensorless control methods for BSM can be divided into the following two types.

1) One is based on the fundamental mathematical model of 
the machine. Literature [10] and [11] proposed an extended Kalman filter for the estimation of the rotor position of BSM in the high speed range where the back-EMF could be measured, and rotor position calculation method is directly determined according to the formulas of the flux linkage model and back-EMF. However, these methods are not applicable for the zero and low speed range of BSM used as the starter for engine cranking, where the back-EMF can't be detected, and the accuracy of the position estimation could be easily affected by the interference of load and speed changes during the starting procedure.

2) The other is the HFSI method based on the saliency effects of the rotor, which has been widely used for low-speed sensorless control and rotor initial position detection for PMSM[12]-[15]. The main generator of BSM is instinctively a salient-pole synchronous machine, thus, In [16], the rotating high frequency voltage signal is injected into the armature windings of the main generator of BSM, and the high frequency current response signal with rotor position information is extracted and further processed to obtain the rotor position. While, certain drawbacks associated with the common sensorless control method employed for BSM in the aircraft starter/generator can be drawn as follow.

a. Since the high frequency mathematical model of BSM is more complex than PMSM due to the field and damper windings in the rotor, plenty of assumption and approximation have to be adopted in the signal processing of the position information extraction, and it affects the accuracy of position detection.

b. The injected high frequency signal must meet certain signal-to-noise ratio requirements, so that its amplitude should be high enough for the response signal detection, then, the torque ripples will be inevitably generated by the injected signals during the main generator in the starting process, and it will deteriorated the output torque performance of BSM, especially for the high-capacity starter/generator.

c. In the conventional approach for the detection of initial rotor position for BSM, the extra voltage signal injection or inductance variation for the pole polarity discrimination have to be involved in the beginning of the rotor position estimation method[17]-[19]. It takes additional time to calibrate the pole polarities and it will deteriorate the torque response speed in the starting control.

d. When BSM is employed as the starter for the engine cranking, the AC excitation control of the main exciter and sensorless starting control of the main generator should be adopted in the starting control, the system control algorithm is very complex to reduce the reliability and robustness of the proposed starter/generator.

Thus, in this paper, a novel integrated method of combining three-phase AC excitation and HFVSI is proposed to solve the above problems without any additional required hardware and winding reconfigurations for BSM in starting mode. In section II, the operation principle of the three-phase AC excitation combined with the $6^{\text {th }}$ harmonic voltage signal injected in the field windings of main generator are proposed, the influence of the nonlinear rectifier for HFSI are analyzed. Section III concentrates the asynchronous demodulation method for the rotor position estimation, and initial rotor position detection by the polarity decision of induced currents of armature windings of the main generator within the excitation establishment process is explained. The HFVSI method for the sesorsless starting control is given in section IV. The excitation effect and accuracy of the position detection algorithm for BSM is validated by the simulation results in section V. Finally, the experiments are implemented based on the BSM starting control platform to prove the validation and effectiveness of the proposed method in section VI, and the conclusion is drawn in section VII.

\section{OPERATION PRINCIPLE}

\section{A. System Configuration of BSM in Starting mode}

Fig.1 shows the schematic diagram of a BSM in starting control mode. The BSM consists of the main generator, main exciter and permanent magnet sub-exciter. The main exciter has a three-phase excitation winding which is different to the traditional three-stage synchronous generator with the single DC excitation winding[20]. In the starting mode, due to the relative low speed range, the output of the sub-exciter is negligible and could not offer the excitation power, thus only the main exciter and main generator are involved in starting control. The three-phase excitation windings of the main exciter are energized directly by the fixed $400 \mathrm{~Hz}$ AC power from the ground power supply or the auxiliary power unit without any excitation controller, and the main exciter works as the rotating transformer to supply the excitation power for the main generator through the rotating rectifier. Although the field current of the main generator is uncontrolled in this direct excitation method, and it will result in the variation of the field current with respect to the rotor speed, while, it could be ignored for the constant output torque of the main generator for the engine cranking in the low speed range.

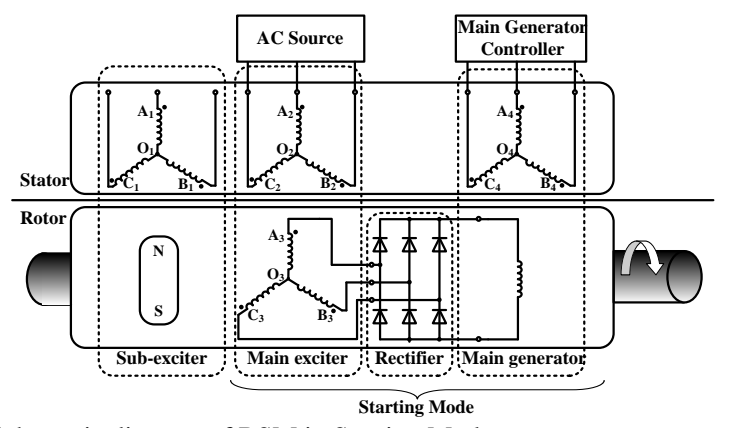

Fig.1 Schematic diagram of BSM in Starting Mode

\section{B. Integrated three-phase AC excitation and High Frequency Voltage Signal Injection (HFVSI)}

The flux equation of the excitation windings of the main exciter, which is similar to the wound rotor induction machine, in dq-reference frame can be shown as

$$
\left[\begin{array}{l}
\psi_{d s} \\
\psi_{q s} \\
\psi_{d r} \\
\psi_{q r}
\end{array}\right]=\left[\begin{array}{cccc}
L_{s} & 0 & L_{m} & 0 \\
0 & L_{s} & 0 & L_{m} \\
L_{m} & 0 & L_{r} & 0 \\
0 & L_{m} & 0 & L_{r}
\end{array}\right]\left[\begin{array}{c}
i_{d s} \\
i_{q s} \\
i_{d r} \\
i_{q r}
\end{array}\right]
$$


Where, $L_{\mathrm{S}}$ is the self-inductance of the stator winding, $L_{r}$ is the self-inductance of the rotor winding, and $L_{m}$ is the mutual inductance between stator and rotor winding. Additionally, $\psi_{d s}$ and $\psi_{q s}$ are the d-axis and q-axis flux of the stator, while $\psi_{d r}$ and $\psi_{q r}$ are the d-axis and q-axis flux of the rotor respectively. Besides, $i_{d s}$ and $i_{q s}$ represent the d-axis and q-axis current of the stator, while $i_{d r}$ and $i_{q r}$ indicate the d-axis and q-axis current of the rotor respectively.

The voltage can be deduced as

$$
\left[\begin{array}{l}
u_{d s} \\
u_{q s} \\
u_{d r} \\
u_{q r}
\end{array}\right]=\left[\begin{array}{cccc}
R_{e s} & 0 & 0 & 0 \\
0 & R_{e s} & 0 & 0 \\
0 & 0 & R_{e r} & 0 \\
0 & 0 & 0 & R_{e r}
\end{array}\right]\left[\begin{array}{l}
i_{d s} \\
i_{q s} \\
i_{d r} \\
i_{q r}
\end{array}\right]+p\left[\begin{array}{l}
\psi_{d s} \\
\psi_{q s} \\
\psi_{d r} \\
\psi_{q r}
\end{array}\right]+\left[\begin{array}{c}
-\omega_{e} \psi_{q s} \\
\omega_{e} \psi_{d s} \\
-\left(\omega_{e}-\omega_{r}\right) \psi_{q r} \\
\left(\omega_{e}-\omega_{r}\right) \psi_{d r}
\end{array}\right]
$$

Where, $u_{d s}$ and $u_{q s}$ are the voltages of the stator winding in dq-axis while $u_{d r}$ and $u_{q r}$ are the voltages of the rotor windings. Besides, $R_{e s}$ and $R_{e r}$ are the resistance of the stator and rotor windings respectively. $\omega_{e}$ denotes the angular frequency of excitation voltage.

The three-phase $400 \mathrm{~Hz}$ AC excitation power can be expressed as $u_{d s}=U_{m}$ and $u_{q s}=0$ by the dq transformation, where $U_{m}$ is defined as the amplitude of the excitation voltage. In the no-load condition, by substituting (1) into (2), the induced voltages of rotor in dq-reference frame are obtained as

$$
\left\{\begin{array}{l}
u_{d r}=\frac{\left(\omega_{e}-\omega_{r}\right) L_{m} U_{m}}{\sqrt{R_{s}^{2}+\left(\omega_{e} L_{s}\right)^{2}}} \sin \varphi \\
u_{d r}=\frac{\left(\omega_{e}-\omega_{r}\right) L_{m} U_{m}}{\sqrt{R_{s}^{2}+\left(\omega_{e} L_{s}\right)^{2}}} \cos \varphi
\end{array}\right.
$$

Where, $\varphi$ is impedance angle of the stator windings. Then, the three-phase induced voltage of the rotor windings by the $\mathrm{AC}$ excitation can be derived by the inverse dq transformation and shown as

$$
\left\{\begin{array}{l}
u_{A r}=U_{1} \cos \left(\left(\omega_{e}-\omega_{r}\right) t-\varphi-\pi / 2\right) \\
u_{B r}=U_{1} \cos \left(\left(\omega_{e}-\omega_{r}\right) t-\varphi-7 \pi / 6\right) \\
u_{C r}=U_{1} \cos \left(\left(\omega_{e}-\omega_{r}\right) t-\varphi+\pi / 6\right)
\end{array}\right.
$$

Where, $u_{A r}, u_{B b}, u_{C r}$ indicate the induced voltage of armature winding of the main exciter respectively, $U_{1}$ represents the amplitude of the induced voltage, and can be calculated as

$$
U_{1}=\frac{\left(\omega_{e}-\omega_{r}\right) L_{m}}{\sqrt{R_{s}^{2}+\left(\omega_{e} L_{s}\right)^{2}}} U_{m}
$$

It can be seen that the induced voltage by the three-phase AC excitation method is proportional to the amplitude of the AC source. Although the induced voltage varies with the respect to difference value $\omega_{e}-\omega_{r}$ with the increasing speed of BSM in the starting procedure, the frequency of the rotor speed, which is nearly less than $5 \mathrm{~Hz}$ in the starting control method, could be neglected compared to the high $400 \mathrm{~Hz}$ frequency of the excitation voltage. Therefore, the constant output excitation voltage could be achieved for main generator in the constant-torque starting for engine from standstill to the low speed range.

Since the rotating rectifier is employed to rectify the output voltage of the main exciter and obtain DC excitation power for the field winding of BSM, the consequent $6^{\text {th }}$ harmonic voltage in the output voltage of the rotating rectifier is naturally generated. Due to the high inductance of the field windings of the main generator, the current ripples caused by the torque ripples in the field current are very low, therefore, there's almost no voltage ripples in the back EMF of main generator, the THD of BSM used as the generator in the aircraft could be lower than $1 \%$. When BSM is employed as the starter, with the same excitation method, the voltage ripples in the field windings of the main generator almost have no effect on the output torque ripples.

For the study of sensorless starting control herein, the $6^{\text {th }}$ harmonic voltage is adopted as the HFVSI into the field windings of the main generator of BSM. Therefore, the three-phase AC excitation and HFVSI could be integrated for the sensorless starting control of BSM. Compared to the traditional method of HFVSI, where high frequency signal is injected into the armature windings of the main generator, and its amplitude should be high enough to meet certain signal-to-noise ratio requirements for the detection of the response signals. Thus, the torque ripples will be inevitably generated by the injected signal during the main generator in the starting process. Therefore, the novel integrated AC excitation and HFVSI method without any extra signals injected into BSM could also eliminate the torque ripples caused by injected high frequency signals, and output torque performance could be improved for BSM in the sensolress starting processing.

\section{Influence of Non-linear Rectifier for HFVSI}

Fig. 2 illustrates the waveforms of output excitation voltages of the rotating rectifier, where the $6^{\text {th }}$ sequence voltage is the major component. The red dot line is the waveform of the output voltage in the no-load condition, the $6^{\text {th }}$ harmonic voltage is proportional to amplitude of voltage ripple which is defined as the total harmonic voltage $\Delta u_{1}$, and it can be calculated as

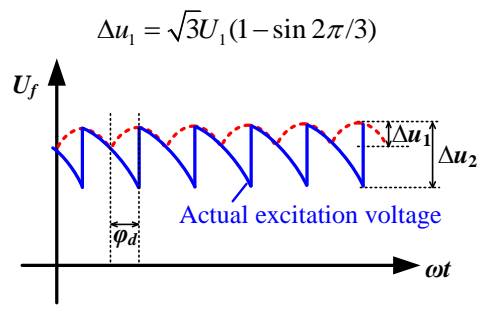

Fig.2 Output excitation voltages of the rotating rectifier

Due to the inductive load of the field winding of the main generator, the overlapping commutation of the diodes will enlarge the amplitude of the total harmonic voltage as the blue solid line shown in Fig. 2 , and total harmonic voltage $\Delta u_{2}$ can be deduced as

$$
\Delta u_{2}=\left\{\begin{array}{lr}
\sqrt{3} U_{1}\left(1-\sin \left(\varphi_{d}+2 \pi / 3\right)\right) & 0<\varphi_{d}<\pi / 6 \\
\sqrt{3} U_{1} \sin \varphi_{d} & \pi / 6<\varphi_{d}<\pi / 3
\end{array}\right.
$$

Where $\varphi_{d}$ is commutation overlap angle. It can be shown that the amplitude of the $6^{\text {th }}$ sequence voltage in the total harmonic voltage for the HFVSI goes higher as the commutation overlap angle increases, and recognition rate of the high frequency response voltage is also raised, it is beneficial to rotor position estimation due to the enlarged $6^{\text {th }}$ harmonic voltage.

However, the acquisition of the output voltage of rotating rectifier is almost impossible due to the rotary condition of the rotor, and the $6^{\text {th }}$ harmonic voltage, which is employed as the 
injected high frequency voltage signal, can't be drawn directly[21]. Moreover, the phase angle of the $6^{\text {th }}$ harmonic voltage also can't be determined due to the nonlinear rotating rectifier and inductive load of the field winding of main generator. Therefore, the traditional synchronous demodulation method for HFVSI based on the accurate high frequency voltage signal for the rotor position estimation is not suitable to be adopted for rotor position estimation of BSM, moreover, the other near-frequency harmonic voltages of the output excitation voltage will also deteriorate the identification of the $6^{\text {th }}$ sequence high frequency voltage signal. Thus, a novel rotor position estimation method should be adopted in the proposed sensorless starting control system of BSM with the integrated three-phase AC excitation and HFVSI.

\section{NOVEL ROTOR POSITION ESTIMATION METHOD}

\section{A. Asynchronous demodulation strategy}

After the high-frequency voltage signal injected into the field winding of the main generator, the responsed high frequency voltages related to the rotor position will be shown in the armature windings, and they could be deduced approximately as

$$
\left\{\begin{array}{l}
u_{A h}=K_{h} u_{f h} \cos \left[\omega_{h} t+\varphi_{h}\right] \cos \theta \\
u_{B h}=K_{h} u_{f h} \cos \left[\omega_{h} t+\varphi_{h}\right] \cos (\theta-2 \pi / 3) \\
u_{C h}=K_{h} u_{f h} \cos \left[\omega_{h} t+\varphi_{h}\right] \cos (\theta+2 \pi / 3)
\end{array}\right.
$$

Where, $u_{f h}, \omega_{h}, \varphi_{h}$ represent the amplitude, angular frequency and phase angle of the $6^{\text {th }}$ harmonic voltage respectively, $K_{h}$ is the coefficient of the induced voltage, $\theta$ is defined as the electrical angle of the rotor position.

The above voltage equation(8) could also be derived by Clarke transformation as follow.

$$
\left\{\begin{array}{l}
u_{\alpha h}=K_{h} u_{f h} \cos \left[\omega_{h} t+\varphi_{h}\right] \cos \theta \\
u_{\beta h}=K_{h} u_{f h} \cos \left[\omega_{h} t+\varphi_{h}\right] \sin \theta
\end{array}\right.
$$

In the conventional synchronous demodulation method, $\cos \theta$ and $\sin \theta$ with the rotor position $\theta$ could be achieved by multiplying sine and cosine curve signal with the same frequency of $\omega_{h}$, then the rotor position could deduced by the phase-locked loop or arctan function of $\sin \theta$ and $\cos \theta$.

While, the high frequency response voltage of formula (8) is an approximate expression where the rotor speed is not considered, as the three-phase main exciter works as the wound rotor asynchronous machine, the angle frequency of the induced voltages in rotor armature windings is not equal to the $400 \mathrm{~Hz}$ due to the slip ratio, therefore, the synchronous demodulation method for the $\cos \theta$ and $\sin \theta$ in equation(9) will not yield result. Furthermore, the near-frequency voltage harmonics due to the non-linear rotating rectifier is abundant, a highly selective filter should be used. It is difficult to select the accurate synchronous sine and cosine signal for the rotor position demodulation.

Therefore, an asynchronous demodulation method of the envelope detection for the high frequency response voltage is proposed in Fig. 3 to decode the rotor position information. The band-pass filter is utilized to filter the irrelevant harmonic voltages of the response voltage due to the non-linear rectifier, and the spindle sharped waveforms of the high frequency voltage response is obtained and shown in Fig.4 (a), where the rotor position is consisted in the envelope curve of the spindle waveform.

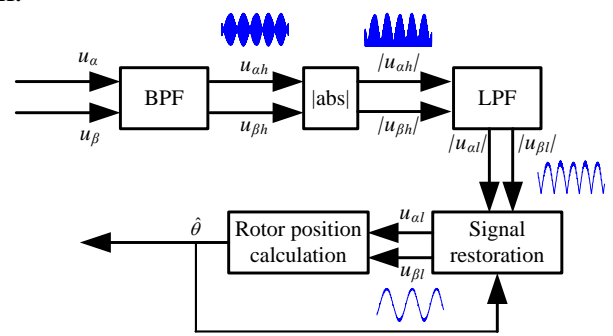

Fig.3 Schematic of asynchronous demodulation method for information decoding of the rotor position

A second-order low-pass filter and a second order high-pass filter are multiplied to obtain a band-pass filter with a pass-band of $2200 \mathrm{~Hz}$ to $2600 \mathrm{~Hz}$ for the extracting of the $2.4 \mathrm{kHz}$ response high frequency voltage. The transfer function is discretized as follow.

$$
G_{B P F}(\mathrm{z})=\frac{0.2039 \mathrm{z}^{3}-0.2706 \mathrm{z}^{2}-0.07065 \mathrm{z}+0.1373}{\mathrm{z}^{4}-2.279 \mathrm{z}^{3}+2.388 \mathrm{z}^{2}-1.244 \mathrm{z}+0.299}
$$

The absolute value module and low-pass filter are employed to obtain the half-wave of the sinusoidal $\left|u_{\alpha l}\right|$ shown in Fig.4 (b). The fourth-order filter as the low-pass filter with a cutoff frequency of $200 \mathrm{~Hz}$ is discretized as bellow. The parameters of the equation(10) and (11) are determined by the filter design tool of the software Matlab.

$$
G_{L P F}(\mathrm{z})=\frac{6.253 \mathrm{z}^{3}-66.23 \mathrm{z}^{2}-6.378 \mathrm{z}+5.584}{\mathrm{z}^{4}-3.813 \mathrm{z}^{3}+5.454 \mathrm{z}^{2}-3.47 \mathrm{z}+0.8282} \mathrm{e}-07
$$

In order to restore the sinusoidal curve of the $u_{\alpha l}$ and $u_{\beta l}$ as shown in Fig.4(b) for the rotor position calculation, the positive and negative half cycle of the envelope waveforms of $\left|u_{\alpha l}\right|$ and $\left|u_{\beta l}\right|$ should be restored and calibrated by initial rotor position, then, $u_{\alpha l}$ and $u_{\beta l}$ corresponding to the rotor position could be obtained to estimate rotor position by phase-locked loop.

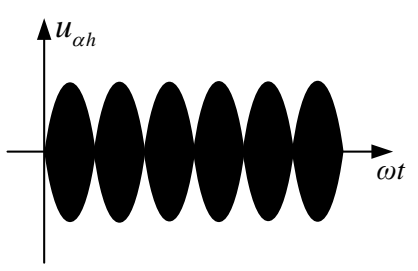

(a) Spindle sharped curve of the output of BPF

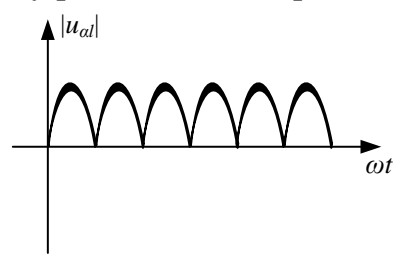

(b) Half-wave sinoidal $\left|u_{\alpha l}\right|$ extracted from LPF
Fig.4 Diagram of the waveforms in the asynchronous demodulation method

As the band-pass and low-pass filters in the asynchronous demodulation method also lead to the phase-lag of the induced high frequency voltage, and the dynamic response process of the phase-locked loop in the starting mode results in the response delay time, over-regulation and deviation of rotor position calculation. Thus, a compensation amount of angle, which is determined by the cutoff frequency of the filters and rotor speed, should be adopted for the calibration of the feedback rotor position angle. Fig.5 shows the block diagram of the signal restoration module, the estimated rotor position $\hat{\theta}$ is employed to recover $u_{\alpha l}$ and $u_{\beta l}$, which could be written as

$$
\left\{\begin{array}{l}
u_{\alpha l}=K_{h 1} u_{f l} \cos \hat{\theta} \\
u_{\beta l}=K_{h 1} u_{f l} \sin \hat{\theta}
\end{array}\right.
$$

Where, $K_{h l}$ and $u_{f l}$ are the coefficient and amplitude of the restored voltage respectively. The initial rotor position and compensation amount is involved in $\theta_{\text {com }}$ for the calibration the 
sine and cosine curve restoration. If the initial rotor position is detected, the ploarties of $u_{\alpha l}$ and $u_{\beta l}$ with respect to the rotor position in the first half-wave period could be determined, $\left|u_{\alpha h}\right|$ and $\left|u_{\beta h}\right|$ are restored to be sine and cosine waves as follow.

1) $u_{\alpha l}$ could be recognize by the comparison of $\hat{\theta}$ and $0.5 \pi$ and $1.5 \pi$. When $\hat{\theta} \leqslant 0.5 \pi$ or $\hat{\theta}>1.5 \pi,\left|u_{\alpha l}\right|$ is equal to the positive half cycle of $u_{\alpha l}$, while $0.5 \pi<\hat{\theta} \leqslant 1.5 \pi$, $\left|u_{\alpha l}\right|$ should multiply -1 to be the negative half cycle of $u_{\alpha l}$.

2) $u_{\beta l}$ is restored by the comparison of $\hat{\theta}$ and $\pi$ homoplastically. When $\hat{\theta} \leqslant \pi,\left|u_{\beta l}\right|$ is the positive half cycle for $u_{\beta l}$, while $\hat{\theta}>\pi,\left|u_{\beta l}\right|$ multiplies -1 to be the negative half cycle of $u_{\beta l}$.

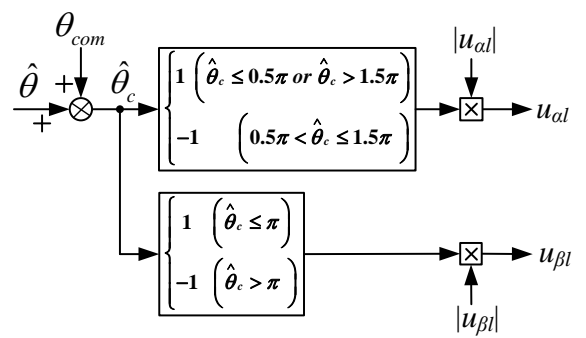

Fig.5 Block diagram of the signal restoration module

Where, the compensation amount $\theta_{\text {com }}$ in Fig.5 is determined by the following two parts, which are coupled together.

1) The phase-angle delay of the BPF and LPF in the signal restoration module, with the speed increasing in the starting processing, the phase lag of the filter will also be increased correspondingly.

2) The hysteresis amount for the polarities decision of the restored $u_{\alpha l}$ and $u_{\beta l}$, which frequency are also increased with respected to the rotor speed, and the detected position of the zero-crossing point will be shifted, moreover, the zero-crossing point of extracted envelope waveform is distorted by the noise interference and the sampling error, therefore, the hysteresis amount should be set to tolerate these variation.

Then, the rotor position $\hat{\theta}$ can be calculated by the following expression in the phase-locked loop shown in Fig.6

$$
u_{\beta l} \cos \hat{\theta}-u_{\alpha l} \sin \hat{\theta}=K_{h 1} u_{f l} \sin (\theta-\hat{\theta})
$$

Where, the amplitude of $u_{f l}$ has no effect on the above signal processing. The corresponding rotor position $\hat{\theta}$ could be estimated and fed back to the signal restoration module repeatedly in the starting processing.

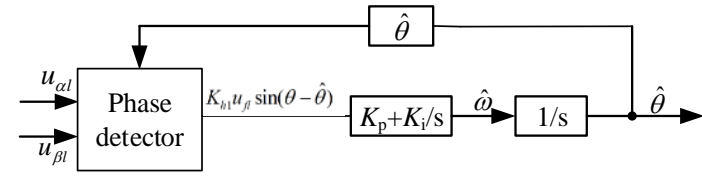

Fig.6 Phase-locked loop for the rotor position calculation

\section{B. Initial rotor position detection}

As explained above, the initial rotor position should be detected firstly as a guarantee that the signal restoration module could work properly for the estimation of rotor position. At standstill, the estimated value of the rotor position may have an error of $\pi$ due to the uncertain polarity of the rotor, which is similar to the PMSM, while, the absolute value module in the above asynchronous demodulation method will also result in the inverse polarity of the restored $u_{\alpha l}$ and $u_{\beta l}$, which will lead to the calculation error of the rotor position.
In order to detect the accurate initial rotor position and improve the property of startup response for the starting control, a combined polarity determination and initial rotor position calibration method for BSM in the excitation current establishment procedure of the main generator is proposed herein. When three-phase windings of the main exciter are energized by the $400 \mathrm{~Hz}$ AC power supply, the excitation current of the main generator gradually increases as a result of the inductance of the field winding, then, the induced voltage will be shown in the armature windings, and it will generate the instantaneous currents due to the short circuit state of zero voltage vector of the SVPWM method for starting control inverter at the standstill status. The polarities of the armature winding currents in $\alpha \beta$-reference frame are corresponding to the location sectors of the rotor shown in TABLE I.

TABLE I

CORRESPONDING RELATION OF THE ROTOR POSITION AND POLARITY OF THE INDUCED CURRENT

\begin{tabular}{cccc}
\hline \hline \multirow{2}{*}{ Sector } & Rotor location range & \multicolumn{2}{c}{ Polarity identification } \\
\cline { 3 - 4 } & & $i_{\alpha}$ & $i_{\beta}$ \\
\hline I & {$[0,0.5 \pi]$} & $\leq 0$ & $\leq 0$ \\
II & $(0.5 \pi, \pi]$ & $>0$ & $\leq 0$ \\
III & $(\pi, 1.5 \pi)$ & $>0$ & $>0$ \\
IV & {$[1.5 \pi, 2 \pi)$} & $\leq 0$ & $>0$ \\
\hline
\end{tabular}

The currents are induced in armature windings of main generator to hinder the change of magnetic flux according to Lenz's law. For different rotor position, the direction of magnitude and induced current varies accordingly, then, the estimated rotor angle could be corrected combined with the sectior judged by the polarities of the $u_{\alpha l}$ and $u_{\beta l}$. When the rotor locates in the range of $[0,0.5 \pi]$, the polarities of $i_{\alpha}$ and $i_{\beta}$ are both negative, and this location range is named as sector I. The other rotor locating sectors of II, III and IV could also be distinguished by the various logic synthesis of the polarities of the induced currents respectively. The current sensors for the current loop control could be employed to identify the current polarities for the discrimination of the pole polarity by the obtained rotor location sectors, then, the determined rotor location could be exploited for the calibration of the initial rotor position, which is calculated from the PLL module.

There are four types of compensations for the initial rotor position $\theta_{0}$ by the polarity decision to the restored $u_{\alpha l}$ and $u_{\beta l}$ at the uncertain rotor position, the estimated rotor angle should be revised according to the polarities of the $u_{\alpha l}$ and $u_{\beta l}$ as follow.

1) If the polarities of the $u_{\alpha l}$ and $u_{\beta l}$ are correct, the initial rotor position $\theta_{0}$ is equal to the calculated value $\hat{\theta}$ from the PLL module. No additional compensation should be added to the estimated rotor angle.

2) If the polarity of the $u_{\alpha l}$ is inversed, while the $u_{\beta l}$ is correct, the initial rotor position $\theta_{0}$ should be calibrated as $\pi-\hat{\theta}$.

3 ) If the polarity of the $u_{\beta l}$ is inversed, while the $u_{\alpha l}$ is correct, the initial rotor position $\theta_{0}$ should be calibrated as $2 \pi-\hat{\theta}$.

4) If the polarities of the $u_{\alpha l}$ and $u_{\beta l}$ are both inversed, the initial rotor position $\theta_{0}$ should be calibrated as $\pi+\hat{\theta}$.

Therefore, based on the above rotor position discrimination and calibration, the initial rotor position detection can be achieved in the field-current establishment of the three-phase $\mathrm{AC}$ excitation. It has the advantages of rapid torque response for sensorless starting control of BSM and no need for the extra voltage sensors and related sampling circuits. 


\section{SENSORLESS STARTING CONTROL METHOD}

Fig.7 shows the schematic diagram of the sensorless starting control of BSM, the current close-loop method with the vector control is employed for the starting control of the main generator, and the angle of the rotor position and three-phase AC excitation are achieved by the proposed integrated method. and a relatively small value of q-axis current is set as command current for the initial rotor position estimation in order to avoid the non-detectable induced voltage of the injected high frequency voltage signal, $u_{\alpha l}$ and $u_{\beta l}$ could be extracted from the output of the current loop and the setup value of the reference current is limited to the certain range to make sure that the BSM could not be driven.

In the initial stage of the starting process, the rotor located sector identification for the initial rotor position is accomplished during the establishment procedure of the excitation magnetic field for the main generator by the three-phase AC excitation, and it is used to calibrate the initial rotor position, which will be differ from the actual position by the fixed value of $\pi$ and $2 \pi$. After the initial rotor position is obtained, the subsequent rotor position estimation link can be operated normally, then, q-axis current should be increased gradually from the predetermined value to the command value in order to avoid the side effect of sudden current change on the rotor position estimation. It can be seen that the complexity of the system structure is obvious reduced compared to the conventional methods, and the successful starting control for BSM could be achieved by the proposed integrated control method without any failure of rotor position estimation.

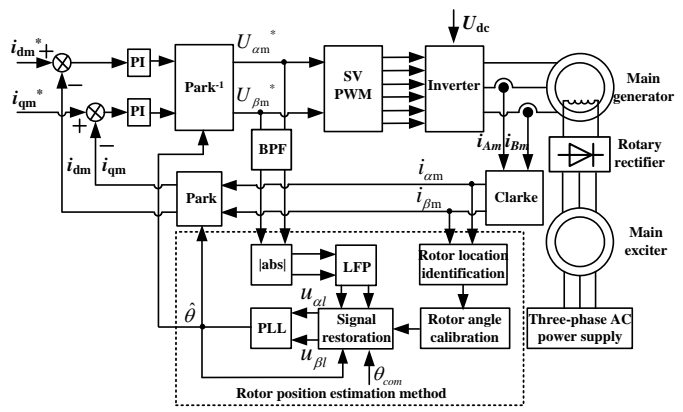

Fig.7 Schematic diagram of the sensorless starting control method for BSM

\section{SiMULATION RESULTS}

The proposed sensorless starting control method is computed from the model of the proposed starter/generator system of BSM with a view to verify the integrated method of three-phase AC excitation and HFVSI by the software of Matlab. The specific parameters of the prototype of BSM in simulation model are displayed in TABLE II and TABLE III.

TABLE II

SPECIFIC PARAMETERS OF THE MAIN EXCITER

\begin{tabular}{lc}
\multicolumn{1}{c}{ Parameter } & Value \\
\hline \hline & \\
Self-inductance of the stator winding $\left(L_{\mathrm{s}} / \mathrm{H}\right)$ & 0.1764 \\
Resistance of the stator winding $\left(R_{e s} / \Omega\right)$ & 4.4 \\
Mutual inductance between stator and rotor winding $\left(L_{m} / H\right)$ & 0.1716 \\
Self-inductance of the rotor winding $\left(L_{\mathrm{r}} / \mathrm{H}\right)$ & 0.1753 \\
Resistance of the rotor winding $\left(R_{e r} / \Omega\right)$ & 4.1 \\
Pole pairs & 3 \\
\hline
\end{tabular}

TABLE III

SPECIFIC PARAMETERS OF THE MAIN GENERATOR

Parameter

Value

Armature winding resistance $\left(R_{S} / \Omega\right)$

Excitation winding resistance $\left(R_{f} / \Omega\right)$

Inductance of d-axis $\left(L_{d} / H\right)$

Inductance of d-axis $\left(L_{q} / H\right)$

20

0.075

Self-inductance of excitation winding $\left(L_{f} / H\right)$

Pole pairs

0.058

5.44

\section{Results of three-phase AC excitation and HFSI}

Fig.8(a) depicts the simulation waveforms of the three-phase $\mathrm{AC}$ excitation, the three-phase field windings of the main exciter are energized by the constant $50 \mathrm{~V} / 400 \mathrm{~Hz}$ AC source, and the induced voltages of the armature windings are distorted due to the load of rotating rectifier and the field winding of the main generator. The mean value of the DC excitation voltage for the main generator with respect to the rotor speed is shown in Fig.8(b), and it maintains constant as $25 \mathrm{~V}$ for the excitation of the main generator. Thus, the constant torque could be achieved for cranking the engine by the current-loop starting control method of BSM.

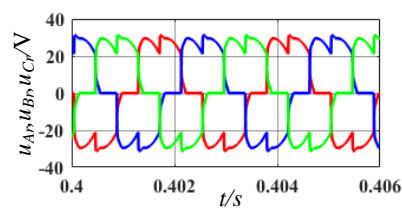

(a) Induced voltage

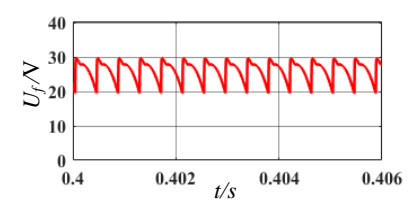

(b) Output excitation voltage
Fig.8 Simulation waveforms of three-phase AC excitation of the main exciter

Moreover, the consequent $6^{\text {th }}$ harmonic voltage of $2.4 \mathrm{kHz}$ utilized as the high frequency voltage signal injected to the field windings of the main generator is consisted in the DC excitation voltage. Fig.9 shows the Fourier analysis waveforms of the DC excitation voltage. Under the no-load condition, the amplitude of the $6^{\text {th }}$ harmonic voltage is $5.846 \mathrm{~V}$. While, the amplitude could increase to be $8.477 \mathrm{~V}$ under the inductive load condition of the field winding, and it is beneficial to the identification of the high frequency response voltage in the armature windings of the main generator for the rotor position estimation, although the near-frequency voltages are abundant.

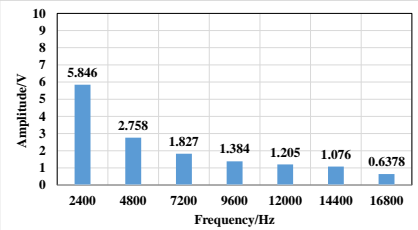

(a) No-load condition

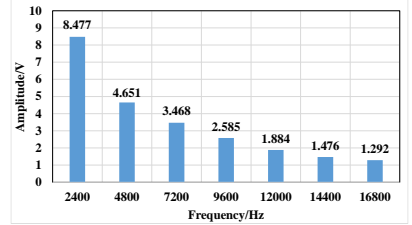

(b) Under load condition
Fig.9 Fourier analysis of the excitation voltages for the field winding of the main generator

\section{Results of asynchronous demodulation method}

Fig.10 illustrates the simulation waveforms of high frequency response voltage. The high frequency response voltages are mixed in the voltages of $u_{\alpha}$ and $u_{\beta}$, and they can be extracted by the band-pass filter, then the spindle sharped waveforms of $u_{\alpha h}$ and $u_{\beta h}$ with the amplitude of $0.8 \mathrm{~V}$ could be obtain in Fig.10(c) and (d).

The simulation waveforms of the absolute values of $\left|u_{\alpha h}\right|$ and $\left|u_{\beta h}\right|$, which are filtered by the low-pass filter with the cutoff frequency of $200 \mathrm{~Hz}$ to eliminate the high frequency harmonic 
voltages, are shown in Fig.11 in the absolute half cycles. Although the phase lag and delay of the zero crossing point will be occurred due to the low pass filter, and the amplitudes of $\left|u_{\alpha l}\right|$ and $\left|u_{\beta l}\right|$ are attenuated, sine and cosine waves of $u_{\alpha l}$ and $u_{\beta l}$ with the signal of the rotor position is restored and corrected by the initial rotor position with the computation angle of $0.02 \mathrm{rad}$ shown in Fig.11(d), then, the rotor position could be calculated by the PLL module.

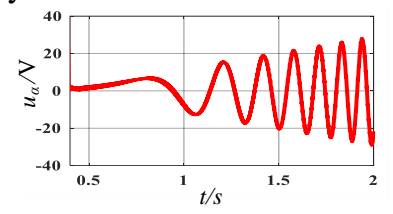

(a) $\alpha$-axis voltage $u_{\alpha}$

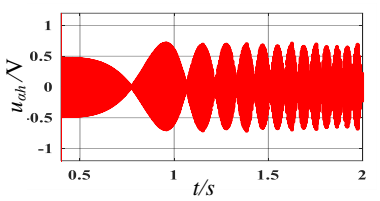

(c) Response voltage $u_{\alpha h}$ Fig.10 Simulation waveforms of the

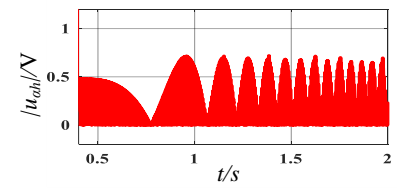

(a) $\left|u_{\text {ah }}\right|$

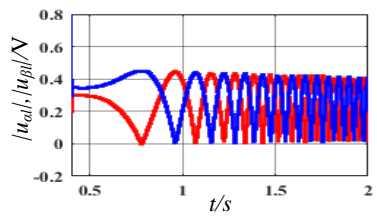

(c) $\left|u_{\alpha l}\right|$ and $\left|u_{\beta l}\right|$

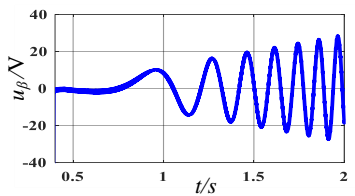

(b) $\beta$-axis voltage $u_{\beta}$

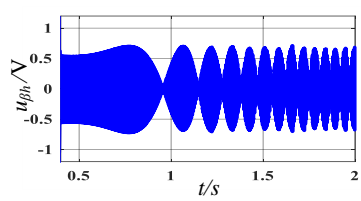

(d) Response voltage $u_{\beta h}$

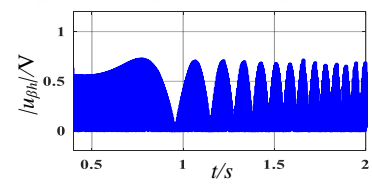

(b) $\left|u_{\beta h}\right|$

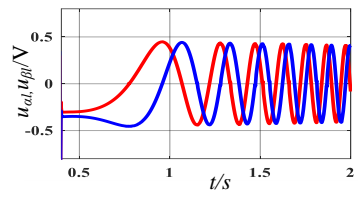

(d) Restored $u_{\alpha l}$ and $u_{\beta l}$
Fig.11 Simulation waveforms of the signal restoration

\section{E. Results of Initial rotor position detection}

Fig.12 shows the induced currents in the establishment of the filed current of the main generator when the rotor locates in the different sectors. The initial rotor position $\theta_{0}=1 \mathrm{rad}$ is in the range $[0,0.5 \pi]$ of the sector 1 , and the polarities of $i_{\alpha}$ and $i_{\beta}$ are both negative. Similarly, when $\theta_{0}$ equals to $2 \mathrm{rad}$, $4 \mathrm{rad}$ and $5.5 \mathrm{rad}$, which are corresponding to the sector II, III and IV respectively, the polarities of the induced $\alpha \beta$-axis currents is consistent with the TABLE 1 described above. The sector determination is accomplished by the functions of $\operatorname{sign}\left(i_{\alpha}\right)$ and $\operatorname{sign}\left(i_{\beta}\right)$ at the delay time of $50 \mathrm{~ms}$ after the three-phase AC excitation, which may take about $200 \mathrm{~ms}$ in the establishment of the field current of the main generator.

As the polarities of $u_{\alpha l}$ and $u_{\beta l}$ are uncertain, the rotor position is calculated by the absolute values of $u_{\alpha l}$ and $u_{\beta l}$ in the proposed asynchronous demodulation method. The estimated value is in the range of $[0,0.5 \pi]$, then, it is revised in four types and matched with the rotor location sector for the accurate initial rotor angle. In the sector 1, the estimated rotor angle is equal to the actual rotor position shown in Fig.13(a), while the rotor is in the sector II, III or IV, the estimated rotor angles could be revised and matched at the time $0.39 \mathrm{~s}$. For the rotor position of $2 \mathrm{rad}$ in the sector II, the estimated rotor angle from the absolute values of $u_{\alpha l}$ and $u_{\beta l}$ is $\hat{\theta}=1.14 \mathrm{rad}$, and the revised value $\pi$ - $\hat{\theta}$ matches to sector 2 shown in Fig.13(b). When the rotor position is 4rad and 5rad corresponding to the sector III and IV respectively, the estimated rotor angles are $0.86 \mathrm{rad}$ and $0.78 \mathrm{rad}$, and they are matched to the sectors in the form of $\pi+\hat{\theta}$ and $2 \pi-\hat{\theta}$. Although there would be overshoot at the tracking point due to the PI regulator of the PLL module, and the accurate rotor position could be achieved for the restoration of $u_{\alpha l}$ and $u_{\beta l}$ in the asynchronous demodulation method.

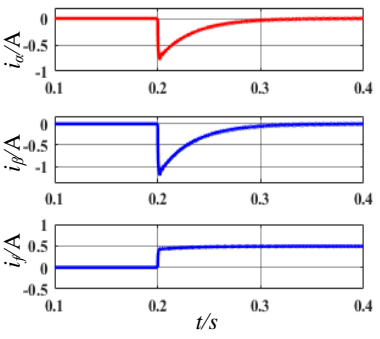

(a) $\theta_{0}=1 \mathrm{rad}$ in sector I
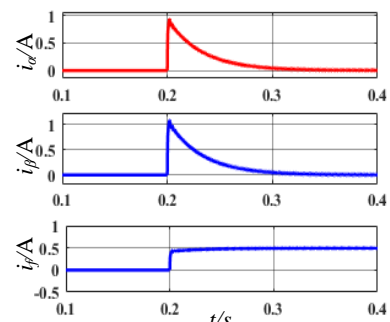

(c) $\theta_{0}=4 \mathrm{rad}$ in sector III

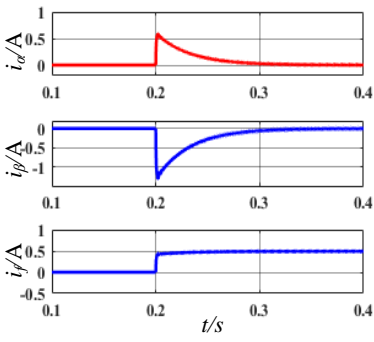

(b) $\theta_{0}=2 \mathrm{rad}$ in sector II
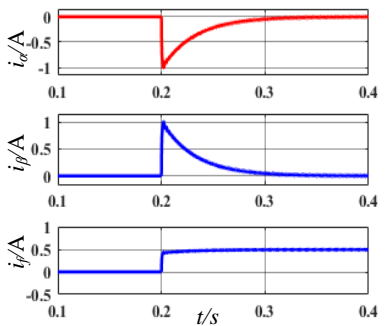

(d) $\theta_{0}=5.5 \mathrm{rad}$ in sector IV
Fig.12 Simulation waveforms of the polarities of induced currents with respect to the sectors

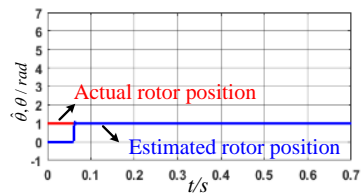

(a) $\theta_{0}=1 \mathrm{rad}$

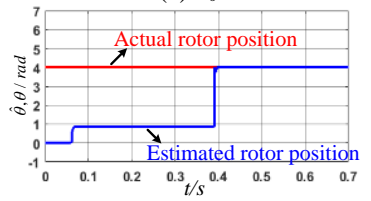

(c) $\theta_{0}=4 \mathrm{rad}$

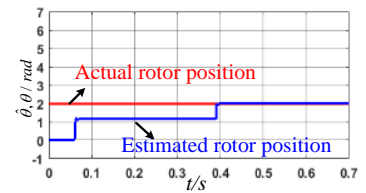

(b) $\theta_{0}=2 \mathrm{rad}$

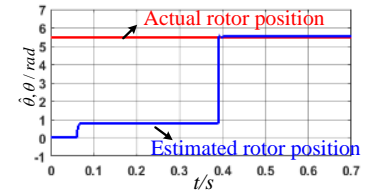

(d) $\theta_{0}=5.5 \mathrm{rad}$
Fig.13 Simulation waveforms of the initial rotor position estimation

\section{F. Results of the sensorless starting control}

From the waveforms of the estimated rotor position in Fig.14(a), it can be seen that the sector location of rotor is recognized in delay time of $\mathrm{t}_{2}=50 \mathrm{~ms}$ during the establishment procedure of the excitation current of the main generator, and it takes about $\mathrm{t}_{3}=400 \mathrm{~ms}$ to estimate the accurate initial rotor position by the proposed method. At the time $t_{1}=0.46 \mathrm{~s}$, the q-axis current of the main generator is gradually increased from $0.4 \mathrm{~A}$ to command value of $10 \mathrm{~A}$ for the constant output torque control for the engine cranking. It can be seen that in the staring processing from standstill to the low speed range, estimated and actual rotor position are well superposed.
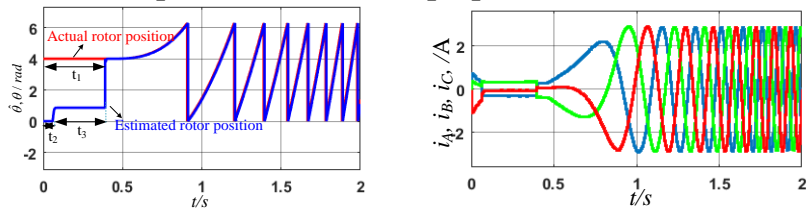
(a) The estimated rotor position

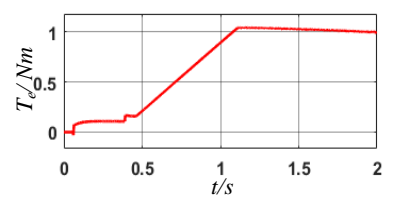

(c) Output torque (b) Three phase currents

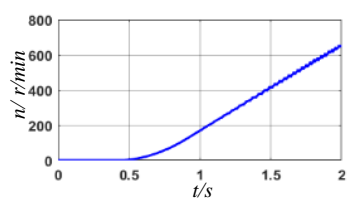

(d) The rotor speed

Fig.14 Simulation waveforms of the sensorless starting control of BSM

The proposed integrated method of three-phase AC excitation and high frequency voltage could be employed in the constant torque starting range from standstill to $650 \mathrm{r} / \mathrm{min}$, from the simulation results, it can be seen that the excellent rotor position tracking could be obtained, the rotor position tracking accuracy can be less than $0.1 \mathrm{rad}$ in the low speed range of 400r/min, although the error of the estimated rotor position in the actual system would be larger than 0.1 rad due to the nonlinear parameters of the generator, rectifier and filters, and the estimated rotor position error is increasing to $0.25 \mathrm{rad}$ with respect to the speed of $650 \mathrm{r} / \mathrm{min}$, the output torque will not decrease significantly shown in Fig.14(c), and the successful constant torque starting control of BSM for engine could be achieved.

\section{EXPERIMENT RESULTS}

The feasibility and practical feature of the proposed integrated three-phase AC excitation and HFVSI method for the sensorless starting control of the BSM have been evaluated using an experiment platform shown in Fig.15.

The controller based on DSP TMS320F28335 and ispXPLD LC5512-MV is established to verify the proposed control strategy. The $2 \mathrm{~kW}$ wound induction machine with the three-phase excitation windings in the stator and rotor is utilized to act as the main exciter of BSM, while the electrically excited synchronous motor is used as the main generator and the encoder is installed for the comparison of the estimated rotor position. The three-phase input ports of rectifier are connected to the rotor windings of the main exciter, and output ports are linked to the field windings of the main generator. Although the rotating rectifier could not be reflected in the platform, the operation principle of the proposed system is nearly similar to the proposed BSM, and integrated method of three-phase AC excitation and HFVSI could be carried on. Moreover, the induced voltage of the main exciter and output voltage of the rectifier could also be detected and displayed to verify the effectiveness of the HFVSI method.

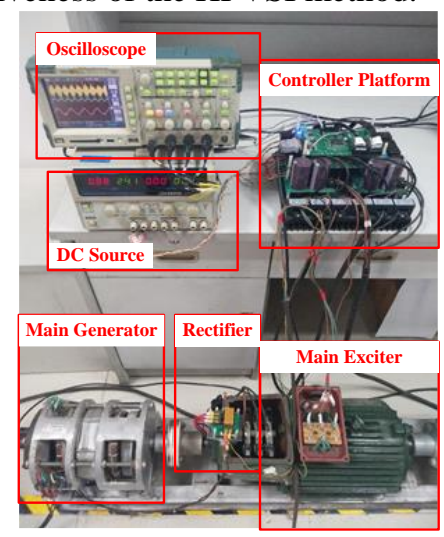

Fig.15 Experiment platform

The three-phase $400 \mathrm{~Hz}$ AC power are energized directly to the stator windings of the main exciter, when BSM is standstill or at low speed range. The induced voltages of the armature windings of the main exciter are distorted due to the no-linear loads of the rectifier and the field winding of the main generator, as shown in Fig.16. The DC excitation voltage with the $6^{\text {th }}$ harmonic voltage determined by the rectifier is maintained as the constant mean value of $25 \mathrm{~V}$, meanwhile, the high frequency voltage of $2.4 \mathrm{kHz}$ is injected to the field winding of the main generator. The induced voltage waveforms are full agree with the simulation results shown in Fig.8.

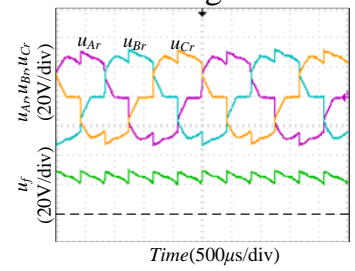

Fig.16 Experimental waveforms of the induced voltage of the exciter and field voltage of the main generator in the three-phase AC excitation method

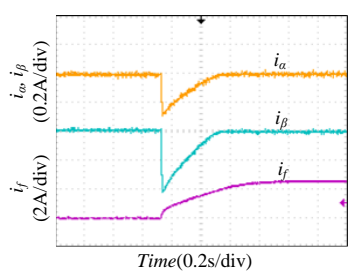

(a) $\theta_{0}=1 \mathrm{rad}$ in sector $\mathrm{I}$

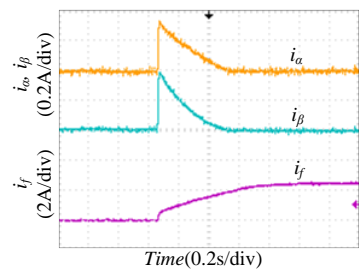

(c) $\theta_{0}=4 \mathrm{rad}$ in sector III

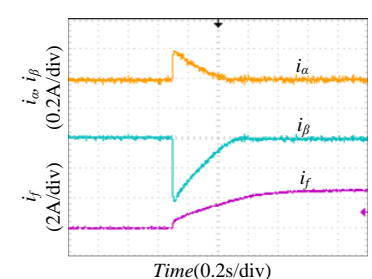

(b) $\theta_{0}=2 \mathrm{rad}$ in sector II

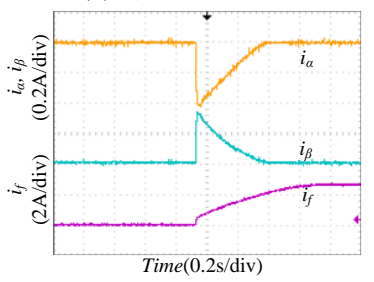

(d) $\theta_{0}=5 \mathrm{rad}$ in sector IV
Fig.17 Experimental waveforms of the induced current in the establishment procedure of the excitation current

Fig.17 illustrates the experimental results of the induced currents in the procedure of establishment of the excitation current, when the initial rotor position is $1 \mathrm{rad}, 2 \mathrm{rad}$, 4rad and 5.5rad respectively corresponding to the sectors from I to IV. The polarities of the $\alpha \beta$-axis currents could be easily identified by the software due to the maximum value is higher than $0.2 \mathrm{~A}$. Although the initial rotor position detection and calibration could be accomplished in the establishment procedure of the excitation current which is about $400 \mathrm{~ms}$ and similar to the simulation results, the actual initial rotor position estimation takes about $1 \mathrm{~s}$ as shown in Fig.18 in order to improve the stability and precision of calibrating processing, where the actual rotor position waveforms are given for the comparison estimation precision of the rotor position.

In each sector, the estimated rotor position could track the actual values rapidly after the calibration processing, small tracking errors are absorbed by the q-axis current, which is setting as $0.4 \mathrm{~A}$ in the control strategy for the response voltage extracting from the output of the current loop. Although the set value of q-axis current could not cranking the BSM, it results in the vibrating of the rotor, and there will be the ripples in estimated rotor position, then it increase the tracking errors of 
the initial rotor position shown in Fig.18. However, this tracking error could still limited to be 0.1rad, and it does not affect the starting control performance of BSM.

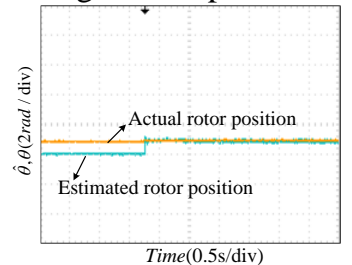

(a) $\theta_{0}=1 \mathrm{rad}$

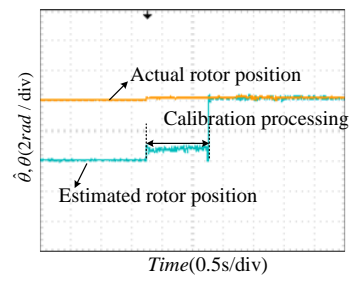

(c) $\theta_{0}=4 \mathrm{rad}$

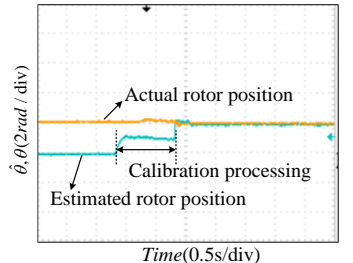

(b) $\theta_{0}=2 \mathrm{rad}$

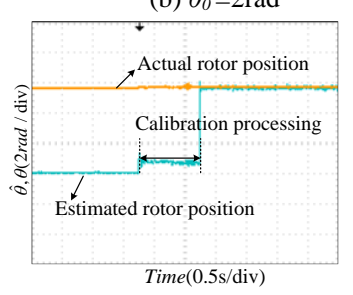

(d) $\theta_{0}=5 \mathrm{rad}$
Fig.18 Experimental waveforms of initial rotor position detection

The waveform of high frequency response voltage $u_{\alpha h}$ is obtained from the output of the BPF, although there are peak voltages in the spindle waveforms, the digital absolute value module and LPF are employed here for the smooth envelope curve, then it is restored to be the sine wave by the phase angle compensation shown in Fig.19, where, the rotor position is consisted in the waveforms of $u_{\alpha l}$ and $u_{\beta l}$, and the sinusoidal waveform of $u_{\alpha l}$ and $u_{\beta l}$ with few harmonics could be calculated by the digital PLL for the rotor position estimation.

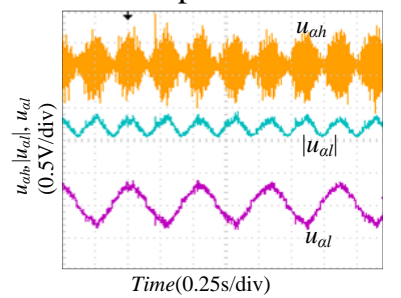

Fig.19 Experimental waveforms of the restoration and correction of the sine and cosine waves

As the speed and acceleration of the starting process increase, the delay effect of the filter becomes more obvious. In particular, the zero-crossing point of extracted envelope waveform is distorted by the noise and interference, it will increase of the rotor position estimation. Thus, the compensation amount is set to be $0.12 \mathrm{rad}$ in the control platform for the signal restoration to meet the demand of the speed range during starting. All the data processing is implemented by the software of DSP, and the intermediate waveforms of these data are illustrated by the oscilloscope through the DA conversion.

Finally, the sensorless starting control of BSM at the low speed range is carried out by the platform. From the comparison waveforms of the actual and estimated rotor position in the starting processing in Fig.20, the initial rotor position detection is accomplished in 1s. Accompanied by the rotor position estimation, the q-axis current of the main generator is gradually increased from $0.4 \mathrm{~A}$ to the command value of $3 \mathrm{~A}$ for the shaft meshing, then, the constant output torque control of by the current-loop control is achieved to crank the engine. From the Fig.21, it can been observed that the estimated rotor position error will be larger with the rotor speed increasing due to the more obvious delay effect of the filters, while, the estimated rotor position error in the starting processing could be limited as $0.135 \mathrm{rad}$ from standstill to the certain speed of $120 \mathrm{r} / \mathrm{min}$ shown in Fig.21, where the back-EMF of the BSM could be conveniently detected for rotor position calculation. Therefore, the proposed integrated method of three-phase AC excitation and HFVSI is verified to be effective in the low speed range for the sensorless starting control of BSM.

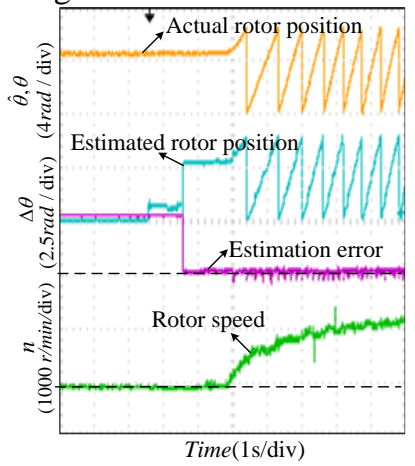

Fig.20 Experimental waveforms of the rotor position detection in the starting procedure

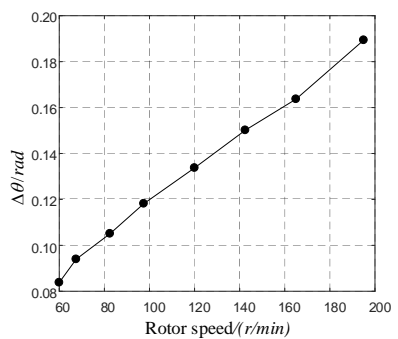

Fig.21 Estimated rotor position error versus rotor speed

\section{CONCLUSION}

In this paper, a novel integrated method for three-phase AC excitation and high frequency voltage injection is proposed for sensorless controlled starting of BSM for the cranking of aero engines. The three-phase AC excitation is accomplished by the direct energization of the constant $400 \mathrm{~Hz}$ AC power to the main exciter, and the consequent $6^{\text {th }}$ voltage harmonics generated by the rotating rectifier are employed as the high frequency voltage injected to the field windings of the main generator, there are no torque ripples generated in the main generator compared to the traditional HFSI methods, the output torque performance of the proposed starting system can also be improved. The novel asynchronous demodulation method is given to estimate the rotor position with high precision of $0.135 \mathrm{rad}$ in the starting processing from standstill to $120 \mathrm{r} / \mathrm{min}$, and the initial rotor position could be achieved effectively by the polarity of the induced currents during the excitation establishment of the main generator for the high torque response performance. From the results of the simulation and experiment, the proposed integrated method does not affect the output torque of the BSM in the starting process, and the proposed method is accomplished effectively and precisely without any additional hardware to that required for the normal starting control of BSM. Furthermore, the proposed sensorless control method could extend the BSM to be the motor in the specific driving applications. 


\section{REFERENCES}

[1] Jie Chang and Anhua Wang, "New VF-power system architecture and evaluation for future aircraft", IEEE Trans. on Aerospace and Electronic Systems, Vol. 42, pp. 527-539, 2006.

[2] Bulent Sarlioglu and Casey T. Morris, "More Electric Aircraft: Review, Challenges, and Opportunities for Commercial Transport Aircraft", IEEE Trans. on Transportation Electrification, Vol.1, No.1, pp. 54-64, 2015.

[3] Zhuoran Zhang, Jincai Li, Ye Liu, Yanwu Xu and Yangguang Yan, "Overview and Development of Variable Frequency AC Generators for More Electric Aircraft Generation System", Chinese Journal of Electrical Engineering, Vol.3, No.2, pp.32-40, 2017.

[4] Zan Zhang, Weiguo Liu, Dongdong Zhao, Shuai Mao, Tao Meng and Ningfei Jiao, "Steady-state performance evaluations of three-phase brushless asynchronous excitation for aircraft starter/generator". IET Electric Power Applications, Vol.10, No.8, pp.788-798, 2016.

[5] Antonio Griffo, Rafal Wrobel, Phil H. Mellor and Jason M. Yon, "Design and Characterization of a Three-Phase Brushless Exciter for Aircraft Starter/Generator". IEEE Trans. on Industry Applications, Vol.49, No.5, pp.2106-2115, 2013.

[6] Dinesh N.Taneja, Hao Huang, Gary A.Padgett, Jan Zywot, J. Paul and Mohamed Abbas, "Dual-Structured Aircraft Engine Starter/Generator", US Patent, 7687928B2, 2010.

[7] Hao Huang, Karipides, Mohame Abbas and David Tsui, "Aircraft Engine Starter/Generator and Controller", US Patent, 2009/0174188 A1, 2009.

[8] Ningfei Jiao, Weiguo Liu, Tao Meng, Jichang Peng and Shuai Mao, "Design and control of a Two-phase Brushless Exciter for Aircaft Wound-Rotor Synchronous Starter/Generator in the Starting Mode", IEEE Trans. on Power Electronics, Vol.31, No.6, pp. 4452-4461, 2016.

[9] Ningfei Jiao, Weiguo Liu, Tao Meng, Jichang Peng and Shuai Mao, "Detailed Excitation Control Methods for Two-phase Brushless Exciter of the Wound-Rotor Synchronous Starter/Generator in the Starting Mode", IEEE Trans. on Industry Applications, Vol.53, No.1, pp.115-123, 2017.

[10] Lahoucine Idkhajine, Eric Monmasson and Amira Maalouf, "Fully FPGA-Based Sensorless Control for Synchronous AC Drive Using an Extended Kalman Filter", IEEE Trans. on Industrial Electronics, Vol.59, No.10, pp. 3908-3918, 2012

[11] A.Maalouf, L. ldkhajine, S. Le Ballois and E. Monmasson, "Field programmable gate array-based sensorless control of a brushless synchronous starter generator for aircraft application", IET Electric Power Applications, Vol.5, No.1, pp.181-192, 2011.

[12] Gaoling Wang, Lei yang, Bihe Yuan, Guoqiang Zhang and Dianguo Xu, "Pseudo-Random High-Frequency Square-Wave Voltage Injection Based Sensorless Control of IPMSM Drives for Audible Noise Reduction", IEEE Trans. on Industrial Electronics, Vol.63, No.12, pp.7423-7433, 2016.

[13] P. L. Xu and Z. Q. Zhu, "Novel Square-Wave Signal Injection Method Using Zero-Sequence Voltage for Sensorless Control of PMSM Drives". IEEE Trans. on Industrial Electronics, Vol.63, No.12, pp.7444-7454, 2016.

[14] Xin Luo, Qipeng Tang, Anwen Shen and Qiao Zhang, "PMSM Sensorless Control by Injecting HF Pulsating Carrier Signal Into Estimated Fixed-Frequency Rotating Reference Frame", IEEE Trans. on Industrial Electronics, Vol.63, No.4, pp.2294-2303, 2016.

[15] Shih-Chin Yang, Sheng-Ming Yang and Jing- Hui Hu, "Design Consideration on the Square-Wave Voltage Injection for Sensorless Drive of Interior Permanent-Magnet Machines", IEEE Trans. on Industrial Electronics, Vol.64, No.1, pp.159-168, 2017.

[16] Antonio Griffo, David Drury, Tadashi Sawata and Phil H. Mellor, "Sensorless Starting of a Wound-Field Synchronous Starter/Generator for Aerospace Applications", IEEE Trans. Industrial Electronics, Vol.59, No.9, pp.3579-3587, 2012.

[17] Riccardo Antonello, Fabio Tinazzi and Mauro Zigliotto, "Benefits of Direct Phase Voltage Measurement in the Rotor Initial Position Detection for Permanent-Magnet Motor Drives", IEEE Trans. on Industrial Electronics, Vol.62, No.11, pp.6719-6726, 2015.

[18] Peilin Xu and Z. Q. Zhu, "Initial Rotor Position Estimation Using Zero-Sequence Carrier Voltage for Permanent-Magnet Synchronous Machines", IEEE Trans. on Industrial Electronics, Vol.64, No.1, pp.149-158, 2017.

[19] X. Zhang, H. Li, S. Yang and M. Ma, "Improved Initial Rotor Position Estimation for PMSM Drives Based on HF Pulsating Voltage Signal Injection", IEEE Trans. on Industrial Electronics, Vol. 65, No. 6, pp. 4702-4713, 2018.
[20] Jiadan Wei, Qingqing Zheng, Mingming Shi, Bo Zhou and Jie Li, "The Excitation Control Strategy of the Three-Stage Synchronous Machine in the Start Mode", Proceeding of Twenty-Ninth Annual IEEE Applied Power Electronics Conference and Exposition (APEC), Fort Worth, USA, pp.2469-2474, 2014.

[21] Ningfei Jiao, Weiguo Liu, Zan Zhang, Tao Meng, Jichang Peng and Yu Jiang, "Field Current Estimation of Wound-Rotor Synchronous Starter-Generator With Asynchronous Brushless Exciters", IEEE Trans. on Energy Conversion, Vol.32, No.4, pp.1554-1561, 2017.

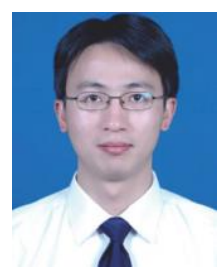

Jiadan Wei (M’10) was born in Danyang, China, in 1981. $\mathrm{He}$ received the B.S. and Ph.D. degrees in electrical engineering from Nanjing University of Aeronautics and Astronautics (NUAA), Nanjing, China, in 2003 and 2009. respectively.Since 2009, he has been a member of the faculty at Department of Electrical Engineering, NUAA, where he is currently an Associate Professor with the College of Automation Engineering, NUAA. From Jul. 2016 to Jul. 2017, he was a visiting scholar in Power Electronics, Machine and Control Group (PEMC) at The University of Nottingham, U.K.

His research interests include aircraft power system, sensorless control of electric machine, and integrated motor and battery charger for electric vehicel.

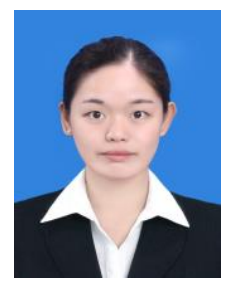

Hua Xue was born in Changzhou, China, in 1996. She received the B.S. degree in electrical engineering from Nanjing University of Aeronautics and Astronautics, Nanjing, China, in 2017, where she is currently working towards the M.S. degree in power electronics and drives. Her research interests include sensorless starting control of motors and their applications in aerospace power systems.

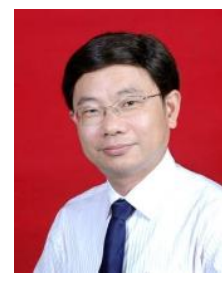

Bo Zhou was born in Wenzhou, China, in1961. He received the B.S. degree from Zhejiang University, Hangzhou, China, in 1983, the M.S. degree from Chongqing University, Chongqing, China, in 1986, and the Ph.D. degree from Nanjing University of Aeronautics and Astronautics (NUAA), Nanjing, China, in 2000. He is currently a Professor with the College of Automation Engineering, NUAA, and the Director of the Jiangsu Key Laboratory of New Energy Generation and Power Conversion. His research interests include power converter, electrical machine driving systems, and renewable power systems.

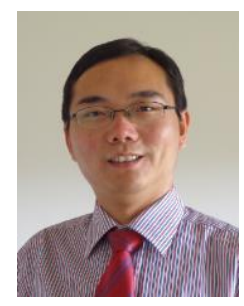

Zhuoran Zhang (M'09-SM'12) received the B.S. degree in measurement engineering and the M.S. and $\mathrm{Ph} . \mathrm{D}$. degrees in electrical engineering from Nanjing University of Aeronautics and Astronautics (NUAA), Nanjing, China, in 2000, 2003 and 2009, respectively. Since 2003, he has been a member of the faculty at Department of Electrical Engineering, NUAA, where he is currently a full professor. From Feb. 2012 to Jun. 2013, he was a visiting professor in Wisconsin Electric Machines and Power Electronics Consortium (WEMPEC), University of Wisconsin-Madison.

His research interests include design and control of permanent magnet machines, hybrid excitation electric machines, and doubly salient electric machines for aircraft power, electric vehicles and renewable energy generation.

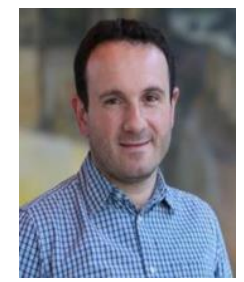

Chris Gerada (M'05-SM'12) received the Ph.D. degree in numerical modelling of electrical machines from The University of Nottingham, Nottingham, U.K., in 2005. $\mathrm{He}$ was a Researcher with The University of Nottingham, working on high-performance electrical drives and on the design and modelling of electromagnetic actuators for aerospace applications. In 2008, he became a Lecturer in electrical machines, in 2011, as an Associate Professor, and in 2013, a Professor at The University of Nottingham. His main research interests include the design and modelling of high performance electric drives and machines. Prof. Gerada serves as an Associate Editor for the IEEE TRANSACTIONS ON INDUSTRY APPLICATIONS and is the Chair of the IEEE Industrial Electronics Society Electrical Machines Committee. 